\title{
Computational studies of history-dependence in nematic liquid crystals in random environments
}

\author{
Amid Ranjkesh, ${ }^{1}$ Milan Ambrožič, ${ }^{1,2}$ Samo Kralj, ${ }^{1,2}$ and Timothy J. Sluckin ${ }^{1,3}$ \\ ${ }^{1}$ Department of Physics, Faculty of Natural Sciences and Mathematics, \\ University of Maribor, Koroška cesta 160, 2000 Maribor, Slovenia \\ ${ }^{2}$ Jožef Stefan Institute, P.O. Box 3000, 1001 Ljubljana, Slovenia \\ ${ }^{3}$ Division of Mathematical Sciences, University of Southampton, Southampton, SO17 1BJ, United Kingdom
}

(Dated: October 23, 2013)

\begin{abstract}
Glassy liquid crystalline systems are expected to show significant history-dependent effects. Two model glassy systems are the RAN and SSS lattice models. The RAN model is a Lebwohl-Lasher lattice model with locally coupled nematic spins, together with uncorrelated random anisotropy fields at each site, while the SSS model has a finite concentration of impurity spins frozen in random directions. Here Brownian simulation is used to study the effect of different sample histories in the low temperature regime in a three dimensional $(d=3)$ model intermediate between SSS and RAN, in which a finite concentration $p<p_{c}$ ( $p_{c}$ the percolation threshold) of frozen spins interacts with neighboring nematic spins with coupling $W$. Simulations were performed at temperature $T \sim T_{N I} / 2$ ( $T_{N I}$ the bulk nematic-isotropic transition temperature) for temperature-quenched and field-quenched histories (TQH and FQH respectively), as well as for temperature-annealed histories $(\mathrm{AH})$. The first two of these limits represent extreme histories encountered in typical experimental studies. Using long-time averages for equilibrated systems, we calculate orientational order parameters and two-point correlation functions. Finite size-scaling was used to determine the range of the orientational ordering, as a function of coupling strength $W, p$ and sample history. Sample history plays a significant role; for given concentration $p$, as disorder strength $W$ is increased, TQH systems sustain quasi-long-range (QLRO) and short-range-order (SRO). The data are also consistent with a long-range order (LRO) phase at very low disorder strength. By contrast, for FQH, only LRO and QLRO occur within the range of parameters investigated. The crossover between regimes depends on history, but in general, the FQH phase is more ordered than than the AH phase, which is more ordered than the TQH phase. We detect also in the QLRO phase a domain-type structural pattern, consistent with ideas introduced by Giamarchi and Doussal [Phys. Rev. B 52, 1242 (1995)] on superconducting flux lattices. In phases in which short-range exponential order occurs, the orientational correlation length in the weak-disorder limit obeys Larkin-Imry-Ma scaling $\xi \sim D^{-2 /(4-d)}$.
\end{abstract}

PACS numbers: 61.30.-v, 61.30.Jf, 61.30.Dk, 61.30.Hn

\section{INTRODUCTION}

It has long been known that the phase diagrams of fluids in finite geometries are shifted as compared to their bulk analogues [1]. Examples of such system are layers (which are effectively two-dimensional), cylindrical pores (effectively one-dimensional) and spherical pores (effectively zero-dimensional). A general term to describe this phenomenon is capillary condensation, although strictly speaking this merely describes the finitegeometry-induced temperature shift in the liquid-vapour transition. Modern liquid-state theory has expended much energy in relating phase boundary shifts to the balance between intermolecular potential energy and surface potential energy [2]. In addition the fluctuation-induced broadening of phase boundaries in reduced-dimensional systems has also been the subject of much attention in the statistical mechanical community [3].

In liquid crystals (LCs), the presence of orientational degrees of freedom, and in particular, competition between surface anchoring and Frank-Oseen elasticity, can complicate this picture. Sheng $[4,5]$, and after him, many other workers (e.g. [6-8]) generalized capillary conden- sation results to the nematic liquid crystal case. Interestingly, in a layer-like pore, the dependence of the phase transition temperature on inverse pore thickness $r^{-1}$ is no longer linear (and dependent on some some combination of surface free energies), but now includes an elasticitydependent $r^{-2}$ term [9]. But experiments on small pores are difficult, precisely because they are small. Typical experiments on LC in pores involves not just a collection, but rather an aggregation, of pores. The system itself will be porous, and although the local geometry of an underlying matrix may be, for example, largely cylindrical, the porous system will often be a continuously connected system in which pores run into each other, and the order parameter in one pore will be, at least weakly, correlated with that of its neighbor.

Thus experiments designed to probe the phase behavior of LCs in individual pores $[10,11]$ necessarily found themselves, at least to some extent, rather probing the behavior of LCs in porous systems. And indeed, such systems carry some extra intrinsic interest with possible technological implications, for field-induced alignment in such materials may carry the potential for diffractionbased rather than polarization-based switchable devices. 
Experimental studies have been carried out in porous systems, using a variety of porous media, a variety of LCs in both nematic and smectic phases, and also using a variety of experimental techniques.

The situation in the mid-1990s was reviewed in the multi-author book edited by Crawford and Žumer [12]. In this paper we confine our interest to the nematic and isotropic phases. Many experiments have been reported using the $n \mathrm{CB}$ series, with $n$ ranging from 5 to 8. Among experimental techniques used have been various types of NMR studies [13-15], magnetically-induced birefringence [16], electro-optics [17], static and dynamic light scattering [18-22], various types of calorimetry [10, 19, 20, 23-28], dielectric spectroscopy [13, 29] and cross-polarized microscopy [25]. Porous systems of a number of different related types have been employed, e.g. anopore-membrane gels [20, 23], silica aerosil dispersions [15, 25, 26, 30-32], membrane filters [22], porous glass [13, 16, 27, 28], solid polymer matrix [17], silicaparticle-based random gel $[14,17,18]$, silica aerogel $[19,21]$ and jammed aqueous laponite suspensions [33]. In general, transitions are rounded, order parameters reduced (with the degree of reduction depending on pore size), and long-range order in the nematic phase is lost.

Much theoretical work on nematic LCs in porous media exploits analogies with so-called random anisotropy magnets (RAM), which are idealized lattice models of amorphous alloys containing rare earth metals. Harris, Plischke and Zuckermann [34] introduced a lattice spin model to describe these systems, which included ferromagnetic interactions $-J \mathbf{m}_{i} \cdot \mathbf{m}_{j}$ between spins $\mathbf{m}$ neighboring sites $i$ and $j$, together with an interaction $-D\left(\mathbf{m}_{\mathbf{i}} \cdot \mathbf{n}_{\mathbf{i}}\right)^{2}$ at each site, with $\mathbf{n}_{i}$ a random unit vector at site $i$ providing a random anisotropy at site $i$ :

$$
\mathcal{H}=-\frac{1}{2} J \sum_{\{i, j\} \mathrm{nn}} \mathbf{m}_{i} \cdot \mathbf{m}_{j}-D \sum_{i}\left(\mathbf{m}_{\mathbf{i}} \cdot \mathbf{n}_{\mathbf{i}}\right)^{2} .
$$

This system provides a textbook example of the importance of dimensionality to the determination of the ground state, and consequently the phase diagram and statistical mechanics, of random systems. Considerable attention has been given to comparison of the Hamiltonian of eq.(1) to spin-glass models [35]. In that case, there is no random field, but rather a random interaction between neighboring sites.

For the HPZ RAM model, an argument due to Larkin [36] and Imry and Ma [37] was used (see e.g. Chudnovsky [38]), but also Pelcovits et al.[39]) to show that for lattice dimension $d$ less than 4 , even for an infinitesimally small degree of randomness $D$, the magnetically ordered (or long-range order LRO) ground state is unstable with respect to the formation of Larkin-Imry-Ma (LIM) domains whose size $\xi \sim D^{-2 /(4-d)}$ (and whose size diverges as $d \rightarrow 4$ ). Thus in physical dimension $d=3$, $\xi \sim D^{-2}$, diverging, as anticipated, as the randomness
$D \rightarrow 0$. Such a (low temperature) phase would possess a two-point orientational correlation function decaying exponentially with distance. High temperature correlations also have this property, and so there would be no discontinuity between low and high temperature properties. The resulting phase is said to possess short-range order (SRO), even though, of course, the relevant length scales are now much larger than the interparticle distance, governing the decay of the high temperature orientational correlations.

An alternative argument, due to Aharony and Pytte [40], suggested that in fact the low temperature state for these systems does not contain LIM domains, but rather quasi-long-range order (QLRO). The magnetic correlations in the low temperature state fall off algebraically, rather than the exponentially as in the LIM case, although the LIM theorem destabilizing the magnetic ground state remains true. Here the magnetic phase transition is preserved in the presence of randomness, although its properties may alter significantly. Other workers have concentrated, particularly in computer simulations, on glassy features of random anisotropy magnets, such as coercivity and remanence [41, 42], noting that in many circumstances, the properties of low temperature phases, although non-magnetic in some formal sense, are nevertheless history-dependent, as in spin-glasses.

The use of spin lattice models to examine general features of the nematic-isotropic transition, and more generally of the nematic LC phase, goes back to work of Lebwohl and Lasher in 1972 [43]:

$$
\mathcal{H}=-\frac{1}{2} J \sum_{\{i, j\} \mathrm{nn}} P_{2}\left(\mathbf{m}_{i} \cdot \mathbf{m}_{j}\right),
$$

where $P_{2}(x)$ is the second Legendre polynomial. The analogy with the HPZ model of (1) (itself a modified version of the exhaustively-studied Heisenberg model) is evident. The lattice model of eq.(2) completely neglects liquid crystalline translational degrees of freedom. However, there is a justification for this apparently negligent step. Fabbri and Zannoni [44] simulated this model using the Monte Carlo method. They measured the magnitude of $\left(T_{N I}-T^{*}\right) / T^{*}$, where $T_{N I}$ is the isotropic-nematic transition temperature, and $T^{*}$ the implied temperature at which the isotropic phase becomes unstable (measured by extrapolating susceptibility data). Whereas the Maier-Saupe mean field theory [45] predicts that this quantity is $\sim 0.1,[44]$ predicts a figure down by a factor of 20 , in very close agreement with experimental measurements by Zink and de Jeu [46].

Two lattice models based on this paradigm have been used to examine nematic LCs in pores. Similar models have also been used in the context of nematic elastomers $[47,48]$. Related free energy models have been examined, using mean field [49-52] and replica trick-renormalization group methods $[47,48,53-56]$. The consensus of the lat- 
ter set of models is that there should be low temperature QLRO, and in particular Feldman [53] has predicted the QLRO exponent to be universal (i.e. independent of the degree of randomness).

The first lattice model is the Random Anisotropy Nematic [49-52, 57-60]. This is the direct Lebwohl-Lasher nematic analogue of the HPZ model:

$$
\mathcal{H}=-\frac{1}{2} J \sum_{\{i, j\} \mathrm{nn}} P_{2}\left(\mathbf{m}_{i} \cdot \mathbf{m}_{j}\right)-D \sum_{i} P_{2}\left(\mathbf{m}_{i} \cdot \mathbf{n}_{i}\right),
$$

where as in eq.(1), the vector $\mathbf{n}_{\mathbf{i}}$ is a random vector at site $i$ of the regular lattice, and all sites are subject to the random anisotropy term.

The second lattice model is the SSS (sprinkled silica spin) model [22, 61-63], in which a proportion $p$ of sites in a Lebwohl-Lasher lattice are frozen:

$$
\mathcal{H}=-\frac{1}{2} J \sum_{\{i, j\} \mathrm{nn}} P_{2}\left(\mathbf{m}_{i} \cdot \mathbf{m}_{j}\right),
$$

subject to a random proportion $p$ of sites $k$ being fixed along (different) random directions $\mathbf{n}_{k}$. Thus, whereas the porosity of the underlying matrix is controlled by the parameter $p$ in the SSS model, it is controlled implicitly by changing the parameter $D$ in the RAN model. Experience in statistical mechanics might suggest, however, that both models reflect some features of the surface-bulk frustration implicit in nematic porous systems, that many general features of their statistical mechanics, both equilibrium and non-equilibrium, would be held in common, and that the field theories which describe long-distance behavior of the two models would likely be very similar. The model discussed in this paper combines features of both the RAN and the SSS models.

Simulation studies of the lattice models are equivocal, perhaps because, as pointed out by Fish and Vink [64], in these random systems lack of self-averaging over randomness can cause problems in data interpretation. In the RAN model, Cleaver et al. [49] found that the low temperature phase of the RAN model, at least for one value of $D$, exhibited QLRO, while Chakrabarti [58] asserted that there was a value of $D$ below which LRO was retained. Extensive simulations $[65,66]$ suggest that LRO is lost even at low $D$ in the analogous magnetic models. The Larkin-Imry-Ma theory in the RAN case [49] suggests that the LRO should always be unstable at least with respect to the formation of domains, with short-range order, and this is in general what has been found in a number of studies on the SSS model [22, 61$63]$.

However, there are history-dependent features at low temperatures in the SSS model. Zero-field cooled (ZFC) and field-cooled (FC) samples behave differently, and the history dependence is related to pinning of disclination lines [63]. Likewise in the simulated HPZ model, the magnetic analogue of the RAN model, quenched samples exhibit LIM domains [65]. However, slowly cooled (annealed) samples possess non-universal QLRO, with the QLRO exponent non-universal (interestingly, in apparent disagreement with the predictions of ref.[53]) and depending continuously on the disorder parameter $D$.

This paper presents a simulation study, using a Brownian relaxation algorithm, which focuses on this history-dependence, where understanding is still extremely patchy. In particular, we investigate the impact of history on nematic structures in a model of a nematic in a porous system. The underlying model, as discussed above, is Lebwohl-Lasher [43] lattice model with particles on sites interacting with nearest neighbors through a traditional second Legendre polynomial interaction. The model (which we specify mathematically in the next section) is intermediate in character between the SSS and RAN models. The randomness in the system is specified thorough randomly distributed impurities of concentration $p$. With each impurity site is associated a randomly distributed local easy axis, and the particle at each such site is constrained to align in the direction of local easy axis. The impurity sites are coupled with neighboring mobile sites through a Lebwohl-Lasher interaction of strength $W$ which may differ from the interaction strength $J$ between the mobile Lebwohl-Lasher sites.

Our paper examines LC orientational ordering properties, over a range of degrees of randomness, as defined by parameters $p$ and $W$, and also over a number of temperatures $T$. As indicated above, we concentrate, although not exclusively so, on the effect of sample history. In this context, "sample history" may indicate whether the system has been cooled quickly ("quenched") or slowly ("annealed"), whether it has been cooled in a field (FC) or in the absence of a field (ZFC), and whether there is some subsequent history (i.e. has the external field been changed/removed and if so has this occurred suddenly or smoothly). The consequence of changing the history can be to change the bulk ordering properties of the sample, which might exhibit, as discussed above, LRO, QLRO or SRO, with in principle possible additional differences in relaxation lengths. Our principal result is to show that, particularly in the weak interaction regime, sample history does indeed impact strongly on the nature of the observed low temperature phase.

The plan of the paper is as follows. The model, and the algorithms that we use to study it, are described in Sec.II. Some cluster mean field arguments, which are necessary to understand the results of our simulations, are developed in Sec.III. The simulation results are presented Sec.IV. In Sec.V we draw some conclusions from our study. 


\section{MODEL}

\section{A. Interaction energy}

The model consists of molecules placed on the points of a three-dimensional simple cubic lattice with nearest neighbor interactions. It is based in general on the LC lattice model of Lebwohl and Lasher [43], and in detail on the RAN [49] and SSS [61] models of LCs in pores. Nematic spins representing LC molecules are placed at a fraction $(1-p)$ of sites. These are free to rotate in the torque field of their neighbors. Frozen nematic spins (impurities) are placed at a fraction $p$ of sites selected at random, and randomly distributed. Lengths in the system are measured in terms of the lattice parameter $a_{0}$. A simulation box contains $N=L^{3}$ sites, where $L$ is the dimension of the box. In production runs we typically set $L=70$, but some of our studies involve comparison of the properties of systems of different sizes [67].

The orientation at lattice site $i$ is denoted by unit vector $\mathbf{S}_{i}$, with, as is usual in discussion of nematic systems, states $\pm \mathbf{S}_{i}$ taken to be physically equivalent. We distinguish between LC molecules, for which $\mathbf{S}_{i} \equiv \mathbf{s}_{i}$ (which we refer to as nematic spins), and the impurities, for which $\mathbf{S}_{i} \equiv \mathbf{m}_{i}$ (which we refer to as impurity spins).

The total interaction energy of the system $\mathcal{H}$, in an external global ordering field $\mathbf{B}=B \mathbf{e}_{B}$, is given as the sum over lattice sites

$$
\mathcal{H}=-\frac{1}{2} \sum_{\{i, j\} \mathrm{nn}} J_{i j}\left(\mathbf{S}_{i} \cdot \mathbf{S}_{j}\right)^{2}-\sum_{i}\left(\mathbf{B} \cdot \mathbf{s}_{i}\right)^{2},
$$

where energy double sums over lattice points are taken over nearest neighbors and the factor of $\frac{1}{2}$ is included to avoid double counting of pair interaction energies. The first term represents the intermolecular interactions. The second term represents the interaction of LC spins with the magnetic field. This is quadratic in field magnitude $B$, and tends to align the LC spins parallel or antiparallel to the field direction $\mathbf{e}_{B}$.

At this stage the sum is over all sites, whether LC or impurity. We now specify that:

(a) $J_{i j}=J>0$ when $\{i, j\}$ are both nematic spins;

(b) $J_{i j}=W$ when one of the spins is a nematic spin and the other is an impurity;

(c) $J_{i j}=0$ when both spins are impurities.

The interaction energy $J$ sets the energy scale for the system. In our calculations, we set this quantity equal to unity. We then measure temperatures $\tilde{T}=\frac{k_{B} T}{J}$, where $k_{B}$ is the Boltzmann constant, $T$ is the absolute temperature, and frozen spin interactions $\tilde{W}=\frac{W}{J}$ in terms of $J$.
Note that local ordering interaction between impurities and LC molecules might replace the high-temperature isotropic phase with the paranematic $(P)$ phase exhibiting a finite degree of orientational ordering. However, in general, the frustration due to the spatial variation of frozen impurities decreases the degree of ordering in the low-temperature orientationally ordered phase. Such strongly frustrated states exhibiting glassy features are commonly referred to as the speronematic $(S N)$ phase [50].

\section{B. Simulation method}

The simulation method involves relaxing nematic spins subject to a Langevin equation with thermal noise. The magnitude of local spins is conserved, and simulations include this normalization constraint $\left|\mathbf{s}_{i}\right|=1$ by adding Lagrange multipliers $\lambda_{i}$ into the functional $\mathcal{H}^{*}$, defined as

$$
\mathcal{H}^{*}=\mathcal{H}+\sum_{i}\left(\mathbf{s}_{i} \cdot \mathbf{s}_{i}-1\right) \lambda_{i}
$$

At a finite temperature equilibrium or metastable states are reached via a Langevin-type relaxation process. We follow the specific technique of Bradač et al. [68], who themselves followed a general method introduced by Ermack and McCammon [69] (see also Dickinson et al. [70] and Ying and Peters $[71,72])$. The LC spins $\mathbf{s}_{i}$ obey a stochastic Langevin equation:

$$
\frac{\partial \mathbf{s}_{i}}{\partial t}=-\frac{D_{r}}{k_{B} T} \frac{\partial \mathcal{H}^{*}}{\partial \mathbf{s}_{i}}+f_{i}
$$

where $D_{r}$ is a rotational diffusion coefficient for spins on the sphere and $f_{i}$ is a stochastic term obeying the thermal noise relation $\left\langle f_{i}(t) f_{j}\left(t^{\prime}\right)\right\rangle=2 D_{r} \delta\left(t-t^{\prime}\right) \delta_{i j}$. The first term then corresponds to the restoring mechanical torque, and the second term is a random torque, whose mean absolute magnitude ensures thermalizations.

We now discretize eq.(7), yielding the following equation for the change of nematic spin components in a time step $\Delta t$ :

$$
\mathbf{s}_{i}(t+\Delta t)=\mathbf{s}_{i}(t)-\frac{2 D_{r} \Delta t}{k_{B} T} \mathbf{R}_{i}(t)+\Delta \mathbf{S}_{T} .
$$

Here

$$
\mathbf{R}_{i}=\frac{1}{2} \frac{\partial \mathcal{H}^{*}}{\partial \mathbf{s}_{i}}=\sum_{j} J_{i j} \mathbf{g}\left(\mathbf{S}_{i}, \mathbf{S}_{j}\right)+\mathbf{g}\left(\mathbf{S}_{i}, \mathbf{e}_{B}\right),
$$

where $\mathbf{g}(\mathbf{a}, \mathbf{b})=\mathbf{a} \cdot \mathbf{b}((\mathbf{a} \cdot \mathbf{b}) \mathbf{a}-\mathbf{b})$ and $\Delta \mathbf{S}_{T}=f_{i} \Delta t$ represents the random thermal fluctuations.

We henceforth set $J=1$ and therefore measure all the quantities in terms of $J$. We introduce a dimensionless time $\Delta \widetilde{t}=D_{r} \Delta t$ and dimensionless temperature 
$\widetilde{T}=k_{B} T / J$. In simulations we set $\Delta \widetilde{t}=0.016$ in order to obtain sensible reference results in the Lebwohl-Lasher nematic model in the absence of disorder [44]. This model exhibits first-order isotropic-nematic $(I-N)$ phase transition at $\widetilde{T}_{c} \sim 1.1$. In subsequent work we suppress the tilde.

Using this method we calculate for given conditions (i.e., concentration of impurities and coupling strengths) the properties of steady-state quasi-equilibria, for which macroscopic properties of the system no longer change with time. These macroscopic states may be true equilibria, or may be metastable states stabilized by relatively high energy barriers. A typical simulation run takes about $2 \times 10^{4}$ sweeps in order to reach a steady state, where within each sweep all nematic spin orientations are updated once.

In order to probe the effect of sample history on structural properties deep in the speronematic phase, we reach a macroscopic state defined by temperature $T$ using three qualitatively different procedures. These involve:

(i) Quenching the system from an isotropic phase to the temperature $T$. We denote this history as a temperature quenched history (TQH).

(ii) Annealing the system to temperature T. This involves gradually decreasing the temperature from the isotropic phase. At each temperature we equilibrate a structure and use its configuration for the starting profile at the next slightly lower temperature. We denote this history as an annealed history $(\mathrm{AH})$.

(iii) At temperature $T$ we start simulations from a homogeneously aligned sample along a symmetry breaking direction $\mathbf{e}_{B}$. This mimics an experimental setup in which the sample is exposed for long period to a strong external ordering field $\mathbf{B}=B \mathbf{e}_{B}$. We denote this history as a field quenched history (FQH).

We refer to these initial configurations as (i) to (iii) respectively. TQH (i) and FQH (iii) represent extreme complementary initial conditions, and are thus expected to manifest extreme properties. The AH history (ii) may be regarded as representing an intermediate situation, and of course also corresponds to common experimental conditions.

\section{Order parameters and correlation functions}

From the steady states of a system we calculate the global tensor order parameter $\mathbf{Q}^{(g)}$, average local tensor order parameter $\mathbf{Q}^{(l)}$ and the two-point orientational correlation function $G(r)=G(\mathbf{r})$. The nematic spin tensor at site $i$ can be defined in terms of the local spins by

$$
\mathbf{q}_{i}=\frac{1}{2}\left(3 \mathbf{s}_{i} \otimes \mathbf{s}_{i}-\mathbf{I}\right) .
$$

The global order parameter tensor describes average global orientational degree of ordering of the system and is the nematic spin tensor averaged over all sites:

$$
\begin{aligned}
\mathbf{Q}^{(g)} & =\langle\mathbf{q}\rangle_{i}=\frac{1}{2}\left\langle 3 \mathbf{s}_{i} \otimes \mathbf{s}_{i}-\mathbf{I}\right\rangle_{i} \\
& =\frac{1}{N} \sum_{i=1, N} \frac{1}{2}\left(3 \mathbf{s}_{i} \otimes \mathbf{s}_{i}-\mathbf{I}\right) .
\end{aligned}
$$

The brackets $\langle\ldots\rangle$ denote spatial averaging, $\mathbf{I}$ is the identity matrix and $\otimes$ stands for the tensor product. In a low temperature system, the mean global scalar order parameter of the system $S$ can be identified with the largest eigenvector of $\mathbf{Q}^{(g)}$ [73].

The local order parameter tensor describing average local orientational degree of ordering of the system is defined as [74]

$$
\mathbf{Q}^{(l)}=\frac{1}{N} \sum_{i=1, N} \frac{1}{N_{n n}} \sum_{j=1, n n} \frac{1}{2}\left(3 \mathbf{s}_{i} \otimes \mathbf{s}_{j}-\mathbf{I}\right)
$$

where the index $j$ runs over all the neighbors (their number is $N_{n n}$ ) of site $i$. The average local scalar order parameter $s$ is defined as the largest eigenvector of $\mathbf{Q}^{(l)}$.

The nematic two-point correlation function $G(r)$ is defined by

$$
G(r)=\frac{1}{2}\left\langle 3\left(\mathbf{s}_{i} \cdot \mathbf{s}_{j}\right)^{2}-1\right\rangle_{r}==\frac{3}{2}\left\langle\mathbf{q}_{i} \cdot \mathbf{q}_{j}\right\rangle_{r}
$$

where $\langle\ldots\rangle_{r}$ is the average over those LC spin-pairs which are separated by the distance $r$, and in principle the average is also taken over different equivalent random spin configurations.

The spatial dependence of $G(r)$ gives information about the nature of the phase. For both LRO and SRO one expects $G(r)$ to decay exponentially with distance toward a saturated value [49]. In our simulations, we fit $G(r)$ with ansatzes

(a)

$$
G^{(0)}(r)=a_{0} e^{-k r}+b_{0}
$$

or

(b)

$$
G^{(1)}(r)=\frac{a_{1} e^{-k_{1} r}}{r}+b_{1}
$$

where $a_{0}, b_{0}, a_{1}, b_{1}, k$ and $k_{1}$ are adjustable parameters. From eq.(13), it is evident that for a sufficiently large system, $b_{0} \sim b_{1} \sim \operatorname{Tr}\left(\mathbf{Q}^{2}\right) \sim S^{2}$. 
Thus, if the phase possesses SRO, it is expected that $b_{0}=b_{1}=0$ while the length scale $\xi=1 / k$ characterizes the size of correlated nematic clusters. A simple mean field theory [49], based on ideas due to Larkin [36] and Imry and Ma [37] suggests that under a random-field perturbation a homogeneous nematic state is unstable with respect to break-up into clusters. The average size of these clusters should scale as $\xi \sim D^{-\frac{2}{4-d}}$, where $D$ is a measure of the strength of the disorder, and $d$ is the space dimensionality. A cluster mean-field-type estimate given in sec III is consistent with this analysis, and for $d=3$ it suggests $\xi \propto 1 /\left(W^{2} p\right)$.

An alternative scenario, observed in our previous study of such a system [49], involves algebraic decay of correlations, or quasi-long-range order (QLRO). We fit these correlations using the ansatz

$$
G^{(2)}(r)=\frac{a_{2}}{r^{\alpha}}+b_{2}
$$

and $a_{2}, b_{2}$ and $\alpha$ are adjustable parameters. One expects $G^{(2)}(r \rightarrow \infty) \rightarrow 0$. However, the decay of correlations with distance is relatively weak, and finite-size effects are thus expected to be important. In particular, some of the present authors found [49], that direct measurement of $\alpha$ and $b_{2}$, even for a large system, could give misleading results. Rather, better estimates for the power law $\alpha$ could be determined using a finite size scaling analysis [73] of $S=S(L)$, using the relationship

$$
S \propto L^{-\gamma}
$$

Specifically [49], for SRO, the observed mean order parameter in a system of $N$ particles would be given by $S \sim 1 / \sqrt{N}=L^{-3 / 2}$, implying $\gamma=3 / 2$ in this case. By contrast, in the case of LRO, the order parameter tends to a finite non-zero value for large system size $L$, and hence $\gamma=0$. QLRO is signalled by intermediate values of $\gamma$, with $\gamma=\alpha / 2$, so long as $\alpha \leq 3$, although if $\alpha \geq 3$, the SRO finite-size scaling result is recovered.

\section{MESOSCOPIC ESTIMATE OF STRUCTURAL AND PHASE BEHAVIOR}

\section{A. Formulation of problem}

In this section we develop estimates of phase and structural LC behavior in presence of impurity spins within the Larkin-Imry-Ma mesoscopic approach [36, 37]. In this picture, randomly distributed impurities produce random-field-type disturbances, with the consequence that the system adopts a domain-type pattern. As discussed in section I, it appears that true equilibria do not follow this scenario, although with some histories it is observed. This picture serves as the reference mean field model for such random field models.
We describe nematic ordering at mesoscopic level in terms of the local uniaxial tensor order parameter $\mathbf{Q}^{(u)}=$ $s(\mathbf{n} \otimes \mathbf{n}-\mathbf{I} / 3)$, with $\mathbf{n}$ the nematic director field. Impurities are homogeneously distributed within the sample of volume $V$ with volume concentration $p=\frac{N_{i m} v_{i m}}{V}$, where $v_{i m}$ determines the volume of an impurity and $N_{i m}$ is the total impurity number inside the volume $V$.

The total free energy is given by [49]

$$
F=\int\left(f_{c}+f_{e}\right) d V+\int f_{i} d S,
$$

where the first two terms are bulk terms, the last term is a surface term describing the interaction at impurity-liquid interfaces, $f_{c}$ is a Landau condensation term representing the local cost of nematic ordering, and $f_{e}$ is an elastic gradient term.

The condensation term is given by

$f_{c}=\frac{3}{2} a\left(T-T^{*}\right) \operatorname{Tr} \mathbf{Q}^{(u) 2}-\frac{9}{2} b \operatorname{Tr} \mathbf{Q}^{(u) 3}+\frac{9}{4} c\left(\operatorname{Tr} \mathbf{Q}^{(u) 2}\right)^{2}$,

where quantities $a, b, c$ are positive material constants and $T^{*}$ stands for the bulk isotropic supercooling temperature. The elastic term favors spatially homogeneous ordering and is approximated by an isotropic gradientsquared term:

$$
f_{e}=\frac{L_{n}}{2}\left|\nabla \mathbf{Q}^{(u)}\right|^{2}
$$

where $L_{n}$ is temperature independent elastic constant. The interfacial term is modelled by

$$
f_{i}=-\frac{W}{2} \mathbf{m} \cdot \mathbf{Q}^{(u)} \mathbf{m}
$$

where $W$ is a (positive) anchoring strength and $\mathbf{m}$ represents the locally preferred orientation, $|\mathbf{m}|=1$. This ansatz locally favors alignment of $\mathbf{n}$ and $\mathbf{m}$. We assume that impurities are essentially homogeneously distributed with concentration $p$ and orientational probability distribution of $\mathbf{m}$ is spatially isotropic.

We note [49] that the LC-impurity interaction perturbs the high temperature isotropic phase, replacing it by a paranematic $(P)$ phase, exhibiting a finite but orientationally varying degree of orientational ordering. Within the Larkin-Imry-Ma picture adopted in this section, the low temperature phase exhibits SRO, but with a locally significant degree of ordering, modulated by a domain-type structure, often referred to as the speronematic $(S N)$ phase [50].

\section{B. Domain pattern}

In the Larkin-Imry-Ma picture, the disorder breaks the speronematic system into domains of characteristic size $\xi$. We now estimate $\xi$ in this phase. 
We first consider the mean free energy of a single domain of volume $V_{d} \sim \xi^{d}$, within which there are $N_{i m}^{(d)}$ impurities, with surface area $a_{i m}$ per impurity. Within each domain the nematic director may be regarded as aligned, with order parameter $s$. The relevant free energy is given by:

$$
\begin{aligned}
\Delta F_{d} \sim & \left(a\left(T-T^{*}\right) s^{2}-b s^{3}+c s^{4}+\frac{L_{n} s^{2}}{2 \xi^{2}}\right) V_{d} \\
& -\frac{1}{3} N_{i m}^{(d)} W s\left\langle P_{2}\right\rangle a_{i m} .
\end{aligned}
$$

where $\left\langle P_{2}\right\rangle=\frac{1}{2}\left\langle 3(\mathbf{n} \cdot \mathbf{m})^{2}-1\right\rangle$ is the mean value of the 2nd Legendre polynomial of $(\mathbf{m} \cdot \mathbf{n})$ within the domain. The final term in eq.(22) comes from evaluating eq.(21) over the domain, while the penultimate term evaluates approximately the effect of the gradient term. The final term in eq.(22) can now be reexpressed in terms of $\xi$ as follows. We first note that in principle the orientational distribution of orientations $\mathbf{m}$ is isotropic. In an infinitely large domain one expects $\left\langle P_{2}\right\rangle=0$. However, the cancellation will not be precise, and according to the central limit theorem, one expects

$$
\left\langle P_{2}\right\rangle \sim\left(N_{i m}^{(d)}\right)^{-1 / 2}
$$

The number of impurities and the characteristic impurity separation $l_{i m}$ are related by

$$
N_{i m}^{(d)} \sim\left(\frac{\xi}{l_{i m}}\right)^{d}
$$

where

$$
l_{i m} \sim\left(\frac{v_{i m}}{p}\right)^{1 / d}
$$

An expression for $\xi$ is obtained by balancing the $\xi$ dependent elastic and interface contributions in $\Delta F_{d}$. For $d=3$ it follows

$$
\xi \sim \frac{9 L_{n}^{2} s^{2}}{4 p W^{2}} \frac{v_{i m}}{a_{i m}^{2}}
$$

On the other hand, the Larkin-Imry-Ma prediction for $\xi$ is given by [49]

$$
\xi \sim D^{-2 /(4-d)}
$$

yielding an expression for the effective disorder strength for $d=3$ :

$$
D \sim \frac{2 \sqrt{p} W a_{i m}}{3 L_{n} s \sqrt{v_{i m}}}
$$

\section{Phase behavior}

We now consider the phase behavior in three dimensions. In our estimates, we neglect temperature dependence of $\xi$. It is convenient to introduce the following new scaled variables:

(i) scaled order parameter $q=s / s_{0}$, where $s_{0}=s(T=$ $\left.T_{N I}, p=0\right)$;

(ii) dimensionless temperature $\tau=\frac{T-T^{*}}{T_{N I}-T^{*}}$, where $T_{N I}=T^{*}+\frac{b^{2}}{4 a c}$ denotes the bulk isotropic-nematic phase transition;

(iii) $\xi_{n}^{(0)}=\sqrt{\frac{L_{n}}{a\left(T_{N I}-T^{*}\right)}}$, the nematic order parameter correlation length calculated at $T=T_{N I}$;

(iv) $d_{e}^{(0)}=\frac{L_{n} s_{0}^{2}}{W s_{0}}$, the nematic surface extrapolation length likewise calculated at $T=T_{N I}$.

Using this scaling, we can rewrite the free energy in eq.(22) in nondimensional form:

$$
\begin{aligned}
\tilde{f} & =\Delta F_{d} /\left(V_{d} a\left(T_{N I}-T^{*}\right) s_{0}^{2}\right) \\
& =q^{2} \tau-2 q^{3}+q^{4}+\frac{\xi_{n}^{(0) 2}}{2 \xi^{2}} q^{2}-q \sigma,
\end{aligned}
$$

where

$$
\sigma=\frac{\sqrt{p}}{3} \frac{a_{i m}}{\sqrt{v_{i m}}} \frac{\xi_{n}^{(0) 2}}{d_{e}^{(0)} \xi^{3 / 2}}
$$

is a nondimensional measure of the degree of disorder.

In the limit of strong enough anchoring, where $\xi \sim$ $l_{i m} \sim\left(v_{i m} / p\right)^{1 / 3}$, and for spherical impurities of radius $r$, one obtains

$$
\sigma \sim \frac{p \xi_{n}^{(0) 2}}{d_{e}^{(0)} r}
$$

It is now possible to link this model to the theory of nematics in a constant external field [75]. This system is governed by an equation identical to that of eq.(29). In this case, so long as $\sigma<\sigma_{c} \equiv 0.5$, the $S N$ - $P$ phase transition is discontinuous, and takes place at critical scaled temperature $\tau_{c}=1+\sigma$. By direct analogy, in this case also the phase transition shift is given by:

$$
\Delta T_{c}=T_{N I}-T_{c} \sim\left(T_{I N}-T^{*}\right)\left(\frac{\xi_{n}^{(0) 2}}{2 \xi^{2}}-p \frac{\xi_{n}^{(0) 2}}{d_{e}^{(0)} r}\right) .
$$

Within this picture, the phase transition temperature thus decreased by the elastic distortions, but increased by the local interactions. However, for $\sigma>\sigma_{c}$, beyond the nematic-paranematic critical point, the $S N-P$ phase transition would be replaced by gradual changes in degree of ordering with increasing $T$. 


\section{SIMULATION RESULTS}

\section{A. Relaxation to steady state}

Our main interest is to study memory effects in a nonergodic regime, in which the steady-state configuration set depends on the history of the sample. We study in detail steady-state properties subject to two extreme cases, represented by the temperature quenched history (TQH) and field quenched history (FQH). We also present a small number of simulations for samples subject to an annealed history $(\mathrm{AH})$, which we regard as an intermediate case.

We confine study to relatively low temperatures (well below $T_{N I}$ ), where the local degree of order is substantial. We first show how quantities of interest approach a steady state. The first two figures show examples of the time evolution of the energy $\mathcal{H}$ (Figs. 1), and of the order parameter $s$ (calculated from $\mathbf{Q}^{(g)}$, see Eq.(11)) (Figs. 2), in each case subject to FQH at $T=0.5$. . In each simulation the simulations continue typically 5000 sweeps after a plateau in $\mathcal{H}$ dependence was reached at a given temperature.

\section{B. Hysteresis}

In section III, we estimated phase behavior for different impurity-LC interaction strengths. Within a LarkinImry-Ma cluster picture, it is expected that a first order transition to a low temperature phase is retained for a low degree of disorder. But for strong enough disorder, this might be replaced by gradual evolution of nematic ordering. In this context, strong enough disorder may be the result of a high proportion of disordered sites, or of strong coupling with a smaller proportion of sites. If the transition to low temperature behavior is first-order, one might expect the behavior of "up" and "down" temperature scans be different, even when the scans are very slow, as a consequence of the necessity to nucleate a new phase. On the other hand, the absence of a first-order transition and a gradual change of properties would only lead to small differences between "up" and "down" temperature scans.

We examine this hypothesis in Figs. 3, in which we plot some representative examples of the evolution of the degree of orientational order as a function of temperature for a set of different impurity concentrations with $L=70, W=1$. In this figure we compare the degree of nematic order in systems which are first annealed in the absence of an external field from a high temperature $(T=1.3)$ down to zero temperature (this is the $\mathrm{AH}$ history discussed above), and then subsequently slowly heated back to the initial temperature. At each stage, the temperature is reduced or increased by 0.02 in scaled units, starting with an equilibrated configuration at the previous temperature. The system is then allowed to relax to a steady state, following the protocol shown in Figs. 1 and 2 .

These systems are expected $[49,53-55]$ to exhibit QLRO; we see confirmation of this expectation below. Thus the absolute value of $s(T)$ is expected also to be $L$-dependent. However, differences between the "up" and "down" values of $s(T)$, or equivalently a peak in $\Delta s(T)=s^{(\text {up })}-s^{(\text {down })}$ (Fig.3b) nevertheless serves as a signal of history-dependent hysteretic effects. The peak in $\Delta s(T)$ seems likely to be related to the first-order transition (which would give a qualitatively similar signature) from an isotropic phase to a speronematic phase predicted in the Larkin-Imry-Ma picture, even though this picture is somewhat simplified.

We observe that as $p$ increases, and disorder strengthens, the $\Delta s(T)$ peak close to the onset of speronematic order becomes less pronounced, corresponding to a less history-dependent system. By $p=0.25$, corresponding to a higher degree of disorder, it has essentially disappeared, corresponding to a smooth onset of local order. However, the present study does not yet determine the exact character of the phase transitions for a given choice of coupling parameters. To investigate this problem, a more detailed finite size analysis would be required.

\section{Phase structure}

We now turn to the nature of the phase and its dependence on system history. As discussed in sections I and II, we may expect, depending on system histories and choice of parameters, phase structures possessing one of SRO, LRO or QLRO to occur. Such phase structures can be detected either from the properties of orientational correlation function $G(r)$, or by a finite-size scaling analysis of the orientational order parameter dependence on system size. In Fig. 4, we show some typical correlation function profiles $G(r)$.

In case of the SRO or QLRO ansatzes, we note the possibility of more than one possible form of $G(r)$ (e.g. eqs.(14), (15), 16)). In the case of SRO, the best fit is given by eq.(14), and we thus henceforth use this form, $G^{(0)}(r)=a_{0} e^{-k r}+b_{0}$, to estimate average SRO domain size. From the $G(r)$ profile we are able in principle to extract the nature of the phase structure. However, in practice, in several cases the quality of the $G^{(0)}(r)$ (i.e. SRO) and $G^{(2)}(r)$ (eq.(16), i.e. QLRO) fits turn out to be comparable. As a result it is difficult to establish the properties of the phase structure conclusively using the correlation function data alone.

As an alternative, we have analyzed the order parameter results of a set of simulations at different sizes, using finite-size scaling procedure, following Eq.(17). In Figs. 5 , we show the results of finite-size-scaling analyses of $S(L)$ for sets of systems corresponding to those shown in Fig. 4. We see strong evidence that, depending on the 

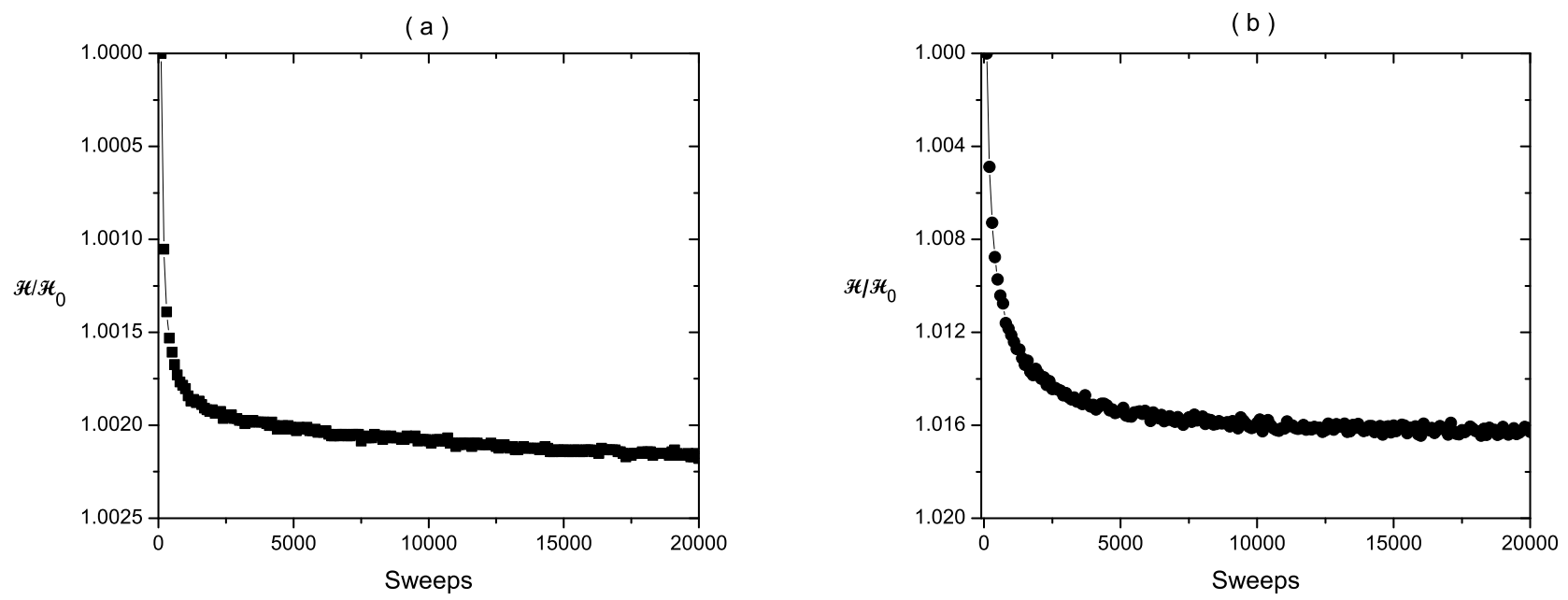

FIG. 1. Examples of time variation of the interaction energy in units of initial isotropic state energy $\mathcal{H}_{0}$, at relatively low temperatures using FQH. In both cases, $p=0.1, T=0.5, L=80$. (a) $W=1.5$, (b) $W=3$.

( a )

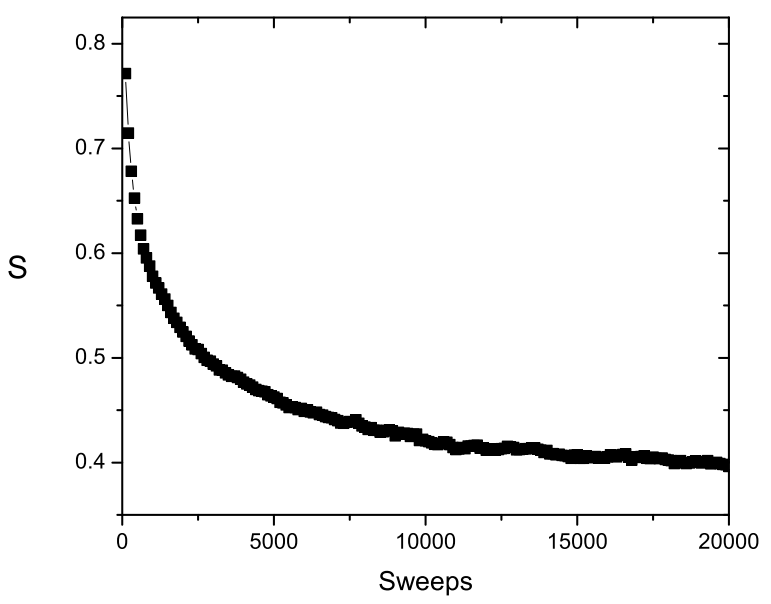

(b)

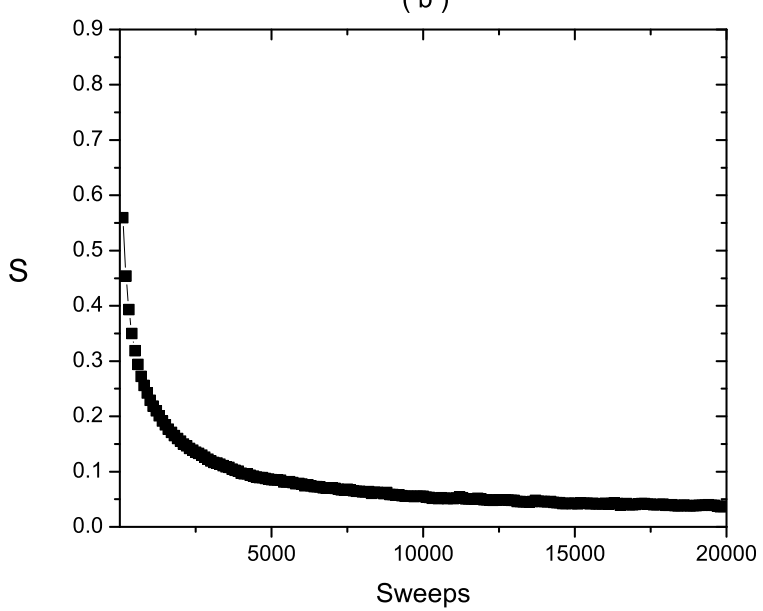

FIG. 2. Typical time variation of degree of local orientational ordering at relatively low temperatures using FQH. In both cases, $p=0.1, T=0.5, L=80$. (a) $W=1.5$, (b) $W=3$.

governing parameters of the system, and the history, all three phase structures (SRO, QLRO, LRO) can occur.

\section{Quenched systems}

We now turn specifically to TQH histories. Previous work with random magnetic models [65] suggests that such quenches may, at least sometimes, cause the resulting phases to exhibit Larkin-Imry-Ma clusters. Furthermore, the mean field analysis of section III suggests a scaling $\xi^{-1} \sim W^{2} p$ in the weak disorder regime. In Figs. 6 and Figs. 7 we present representative $G(r)$ profiles at
$T=0.5$ for different values of $p$ and of $W$.

In both cases, it is apparent that for very weak coupling (i.e. low $p$ or low $W$ ), the analysis indicates that the systems may be exhibiting QLRO or even LRO. But for higher degrees of disorder, the concave form of the loglog plot strongly suggests SRO, with the range of the order decreasing as the degree of disorder (either $p$ or $W)$ increases.

\section{E. Field-cooled systems}

We now consider structures obtained from fieldquenched histories. Some representative profiles at $T=$ 
( a )

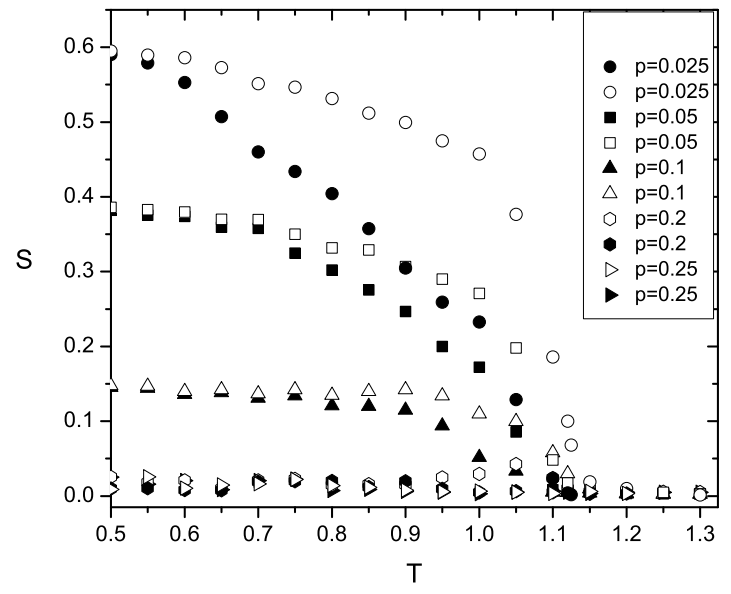

(b)

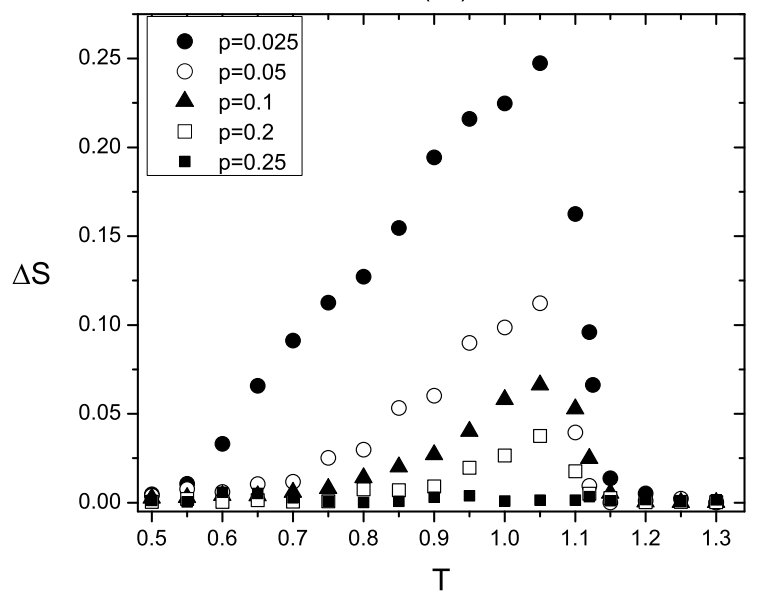

FIG. 3. History-dependence in annealed (AH) systems. (a) Order parameter temperature-dependence $s(T)$. "Up" (empty symbols) corresponds to increasing $T(0 \leq T \leq 1.3)$. "Down" (full symbols) corresponds to decreasing $T(1.3 \geq T \geq 0)$ (see text). In all plots $W=1, L=70$. (b) Behavior of $\Delta s(T)=s^{(u p)}-s^{(\text {down })}$ (see discussion in text).

0.5 and $p=0.1$ for different values of $W$ are depicted in Fig. 8. One sees that in this case either apparent LRO or QLRO is obtained depending on values of $p$ and $W$. For the case shown $(p=0.1)$ LRO seems to prevail for $W \lesssim 0.1 \pm 0.05$, although this may be a finite-size effect. Above this regime QLRO obtains over the whole remaining regime studied.

\section{F. Comparison of histories for specific systems}

In the systems which exhibit QLRO, we have carried out a finite size scaling analysis, This permits $\gamma$ (see Eq.(17)) to be inferred. In the case of SRO, theory suggests that $\gamma \equiv 1.5$. We suppose that fitting errors may intrude, for in some cases, our inferred values suggest $\gamma \geq 1.5$, but it seems likely that this too indicates SRO.

In Fig. 9 we compare the results of TQH (Subsec. IVD), FQH (Subsec. IVE) and some extra AH simulations. For comparison purposes, these simulations use the same set of parameters, using $T=0.5, p=0.1$ and a range of $W \in[0.4]$. In general, as might be expected, where there are differences between samples with different histories, the TQH samples are less ordered than the $\mathrm{AH}$ samples, which in turn are less ordered than the FQH samples.

For very low values of $W \leq 0.1$, the order parameter results alone could be consistent with long-range order for the $\mathrm{AH}$ and $\mathrm{FQH}$ samples. However, the margin of error does allow these systems to have $\gamma \neq 0$ for $W \neq 0$. Our results are not sensitive enough to detect in this case whether there is a critical $W_{c}(p)$ such that LRO order holds for $W<W_{c}$. For $W=0$, these systems should exhibit an equilibrium low temperature long-range order phase, which seems likely to be accessible via a fieldquench or an annealing process.

The key result is that although each type of history seems to indicate QLRO for small $W$, the precise correlation power law seems to depend on the nature of the history. The annealed $(\mathrm{AH})$ and temperature-quenched (FQH) histories give rise to similar behavior, except at very low values of $W$, for which, unsurprisingly, the annealed system is more ordered (i.e. $\gamma$ smaller). At this value of $p$, for strong enough values of $W$, only shortrange order (SRO) remains, but the critical value of $W$ required to destroy the algebraic order is $W \sim 2$ for the temperature-quenched system, but closer to $W \sim 5$ for the field-cooled system, with the annealed system somewhere in between.

\section{G. Domain-type order}

Our finite scaling analysis suggests that for $W>0.1$ QLRO is established for FQH. On the other hand for TQH we obtain either QLRO or SRO. However, fitting $G(r)$ dependencies by using Eq.(14) or Eq.(16) yields comparable quality of fits. This suggests that structural properties of systems exhibit some intermediate structural profiles (with respect to ansatzs Eq.(14) and Eq.(16)). For this purpose we henceforth estimate the dependence of the correlation length $\xi$ on $W$ and $p$ by using Eq.(14) for both hystories in the regime $W>0.1$. The results are shown in Fig. 10. For ease of presentation, we fit the quantity where $k=1 / \xi$. In order to include a wide range of $\{W, p\}$ in this plot, even when other evidence suggests a QLRO phase, we have estimated domain sizes by fitting calculated $G(r)$ profiles using ansatz 
(a)

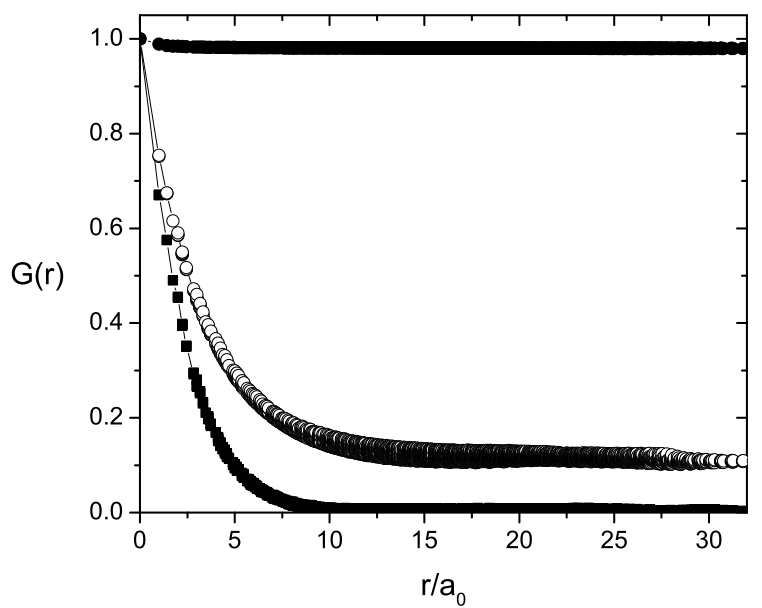

(b)

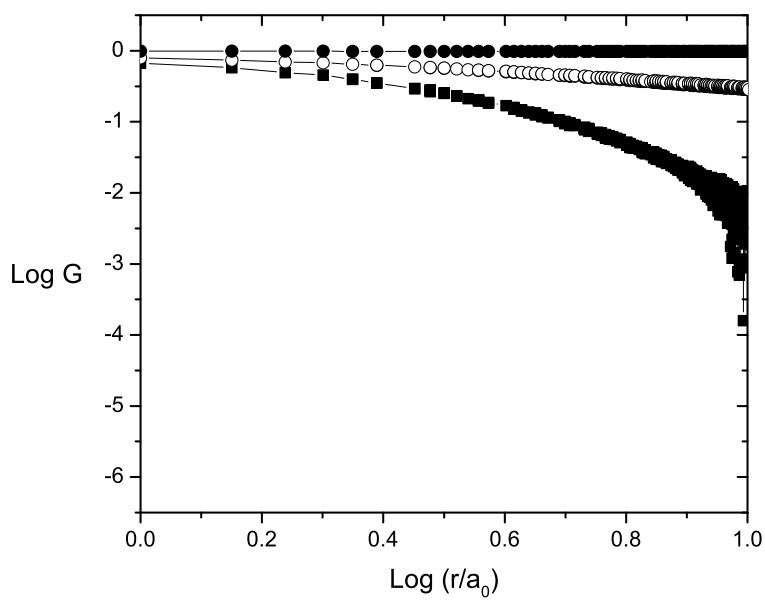

FIG. 4. Examples of orientational correlation functions $G(R)$, demonstrating SRO, QLRO and LRO behavior. (a) $G(R)$ plot. (b) Corresponding Log-Log plots, showing linear decay in the case of QLRO. Legend: SRO (squares, with disorder parameters $W=3, p=0.1$ subject to TQH). QLRO (empty spheres, with disorder parameters $W=1.5, p=0.1$, subject to FQH). LRO (full spheres, with disorder parameters $W=0.01, p=0.2$, subject to FQH). For all simulations, $T=0.5, L=70$.
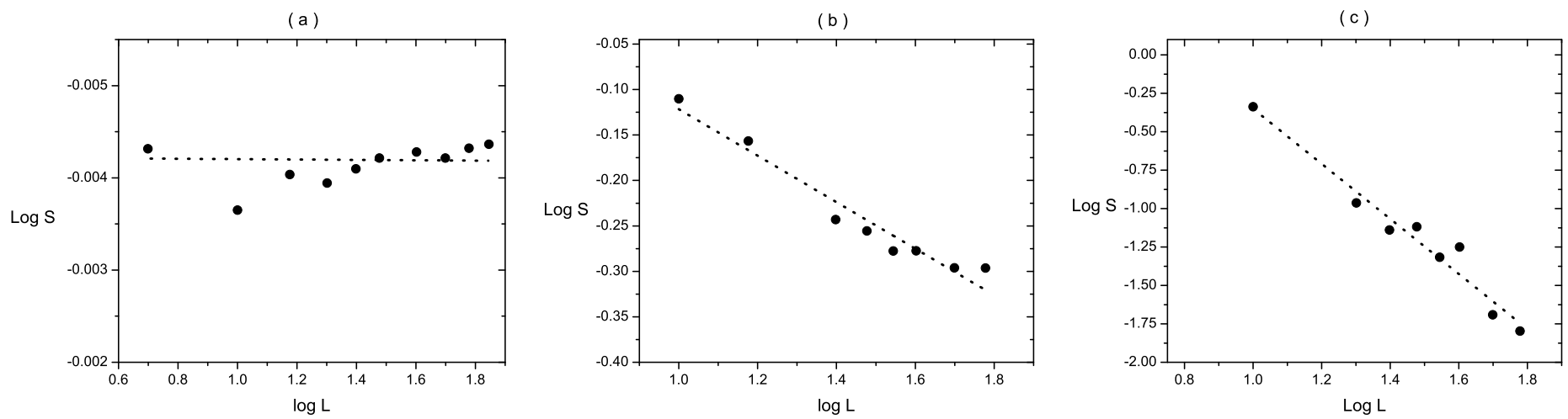

FIG. 5. Finite size analysis of structures for which $G(r)$ is calculated in Fig. 4. Dashed curves are the fits using Eq.(17). (a) LRO: $\gamma=0 ; W=0.01, p=0.2(\mathrm{FQH})$. (b) QLRO: $\gamma=0.25 \pm 0.025 ; W=1.5, p=0.1$ (FQH). (c) SRO: $\gamma=1.75 \pm 0.15$; $W=3, p=0.1(\mathrm{TQH})$. All simulations performed at $T=0.5$. 
( a )

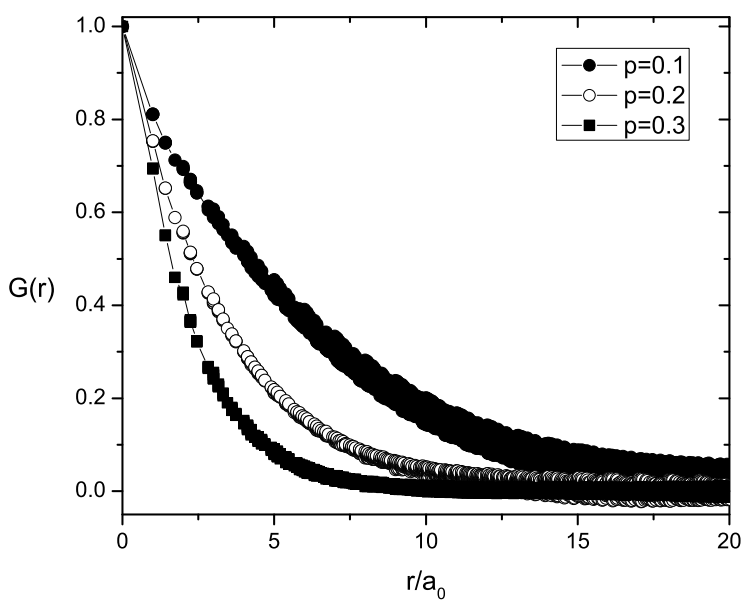

(b)

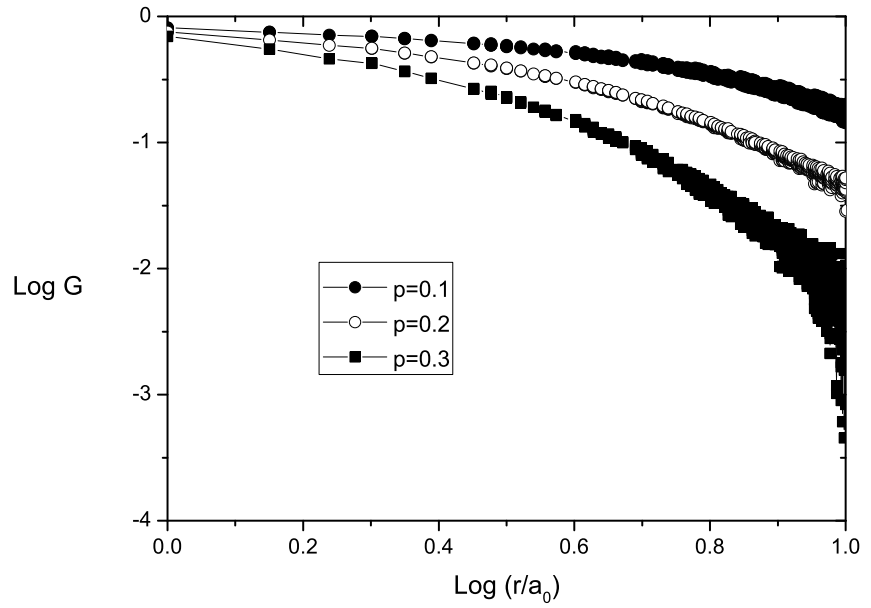

FIG. 6. Variation of $G(r)$ as a function of impurity concentrations $p$ for quenched systems (TQH), at constant impurity strength $W=1(T=0.5, L=70)$. (a) natural plots; (b) log-log plots.

( a )

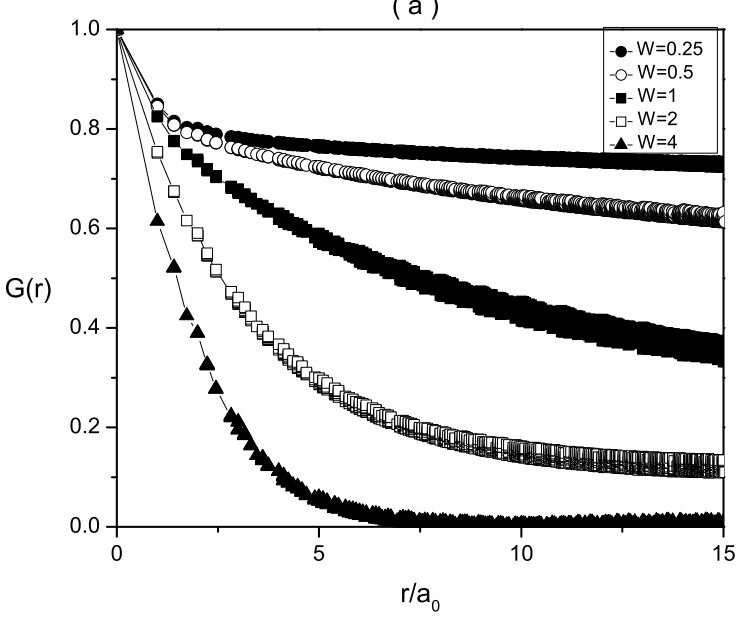

(b)

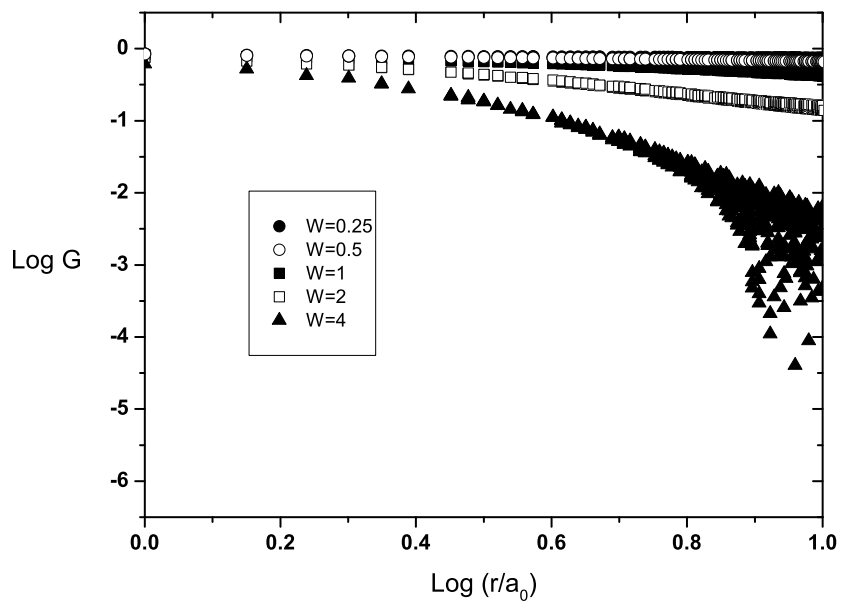

FIG. 7. Variation of $G(r)$ as a function of impurity strength $W$ for quenched systems (TQH), at constant impurity concentration $p=0.1(T=0.5, L=70)$. (a) natural plots; (b) log-log plots.

Eq.(14).

An important result is that FQH and TQH yield comparable value of $\xi$ for a given set $\{p, W\}$. This suggests that size of imprinted domains are comparable for any history. However, the average alignment of domains does depend on the history.

Note that also in case of QLRO fingerprint of a domaintype pattern might be apparent as already suggested by Giamarchi and Doussal [76]. Within each domain correlations between nematic spins are apparently stronger. However, in the case of QLRO the structural crossover region between neighboring domains is smoother in comparison with the SRO case. Estimated values of $\xi=1 / k$ extracted from fits using Eq.(14) are plotted in Fig. 10a (full symbols) and Fig. 10b.

Fig. 10b, shows that in the low disorder regime, the plots of $\xi$ against $W$ for different $p$ collapse closely (although not exactly) onto a single plot if plotted against $p W^{2}$, in agreement with the predictions of eqs.(27),(28). For higher disorder, the average domain sizes saturate. The saturation does not follow a universal law. We comment that in any case we expect that in three dimensions, in any event we expect that $\xi \geq p^{-1 / 3}$, or equivalently, that domain sizes will not be smaller than the distance between impurities, and therefore universality would be lost for higher $p W^{2}$. 
( a )

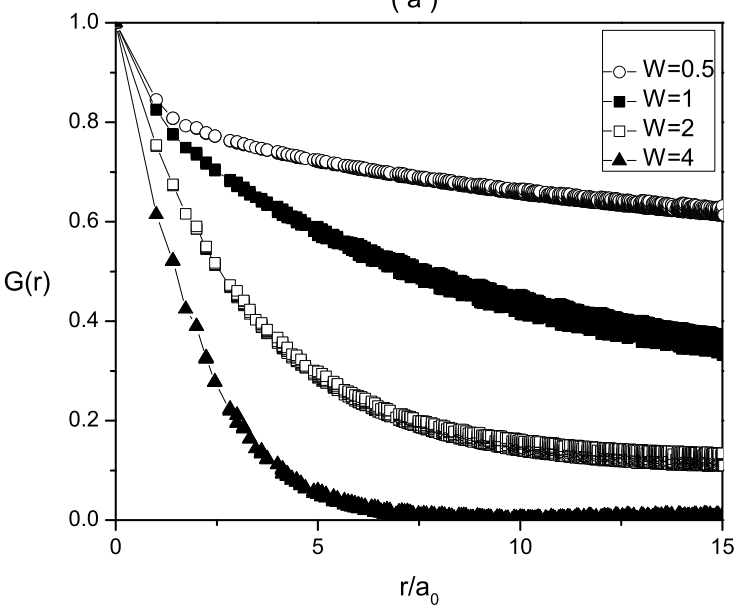

(b)

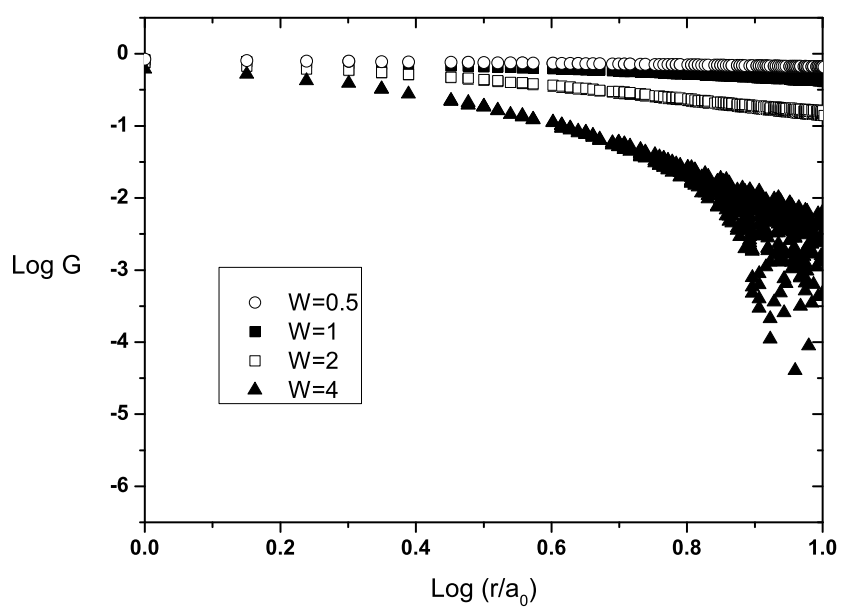

FIG. 8. Field-quenched histories. $T=0.5, p=0.1, L=70$. plots.

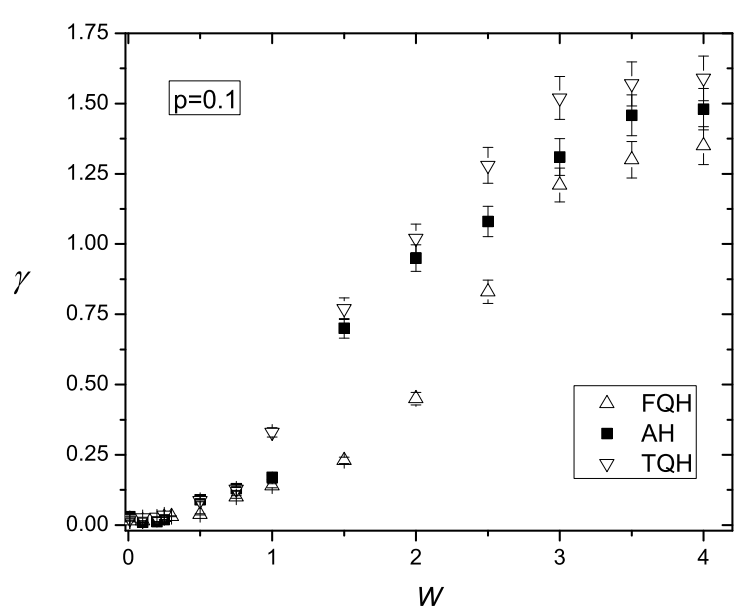

FIG. 9. Comparison of phase structures derived by FQH (triangles), AH (full squares) and TQH (reversed triangles) for $T=0.5, p=0.1$ and a range of $W$. Nature of order indicated by value of $\gamma$ obtained from finite size scaling using Eq.(17). LRO is signaled by $\gamma=0$ confined to very low $W$. QLRO $0 \leq \gamma \leq 1.5$ occurs for intermediate $W$ for TQH and is stable in the whole regime for FQH. SRO occurs at higher $W$ in TQH. Structural characteristics for AH are between those obtained for FQH and TQH.

We note also that if LRO is turned off smoothly (for whatever reason) as $W \rightarrow 0$, then one expects that $\lim _{W \rightarrow 0} \xi^{-1}=0$. However, in Fig.10a in this limit one observes a finite value of $\xi(W \rightarrow 0)^{-1}$ which increases with $p$. The reason behind this is initial sharp decay in $G(r)$ dependence in case of LRO for finite temperatures. In this case $1 / k$ measures order parameter correlation length (a) $G(r)$ for different strengths $W$. (b) Corresponding Log-Log $\xi_{n}$, which is always finite. With increasing $p$ effective interactions strength among nematic spins are weakened due to dilution $\left(\xi_{n} \propto \sqrt{L_{n}}\right.$ in terms of our mesoscopic model, see Eq.(20)). Consequently, with increasing $p$ the correlation length is decreasing and $k$ increasing as observed in Fig. 10a.

\section{CONCLUSIONS}

We have studied the effect of random-field (RF)-like disorder on orientational LC ordering using a LebwohlLasher lattice model approach. The models involves a proportion $(1-p)$ of coupled nematic spins, perturbed by interaction with impurity spins at concentration $p<p_{c}$, with $p_{c}$ the percolation threshold. The impurity spins are randomly placed with isotropic orientational probability distribution, and the nematic-impurity interaction strength is measured by the dimensionless coupling constant $W$. The time evolution of the nematic spins has been simulated using Brownian dynamics, involving a Langevin-type relaxation subject to thermal noise.

This random lattice system is designed to mimic liquid crystals placed in a porous medium. However, we postpone to future work the interesting question of the relation between the topology and geometry of the underlying porous medium and our model parameters. In general, both the amorphous nature of the porous medium, and lattice models with random pinned impurities, are expected to exhibit glassy behavior, with the equilibrium manifold breaking up into many inequivalent isolated submanifolds, and the existence of strongly history- 


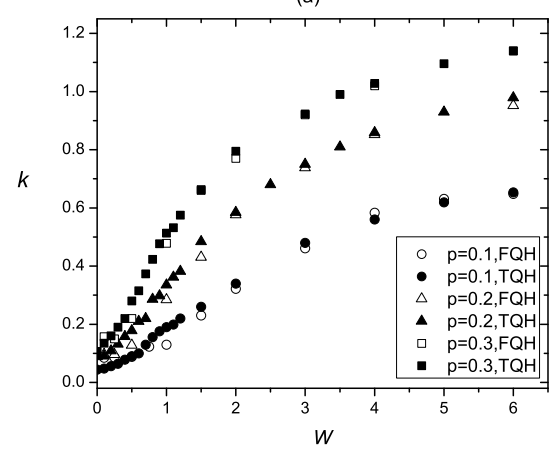

(b)

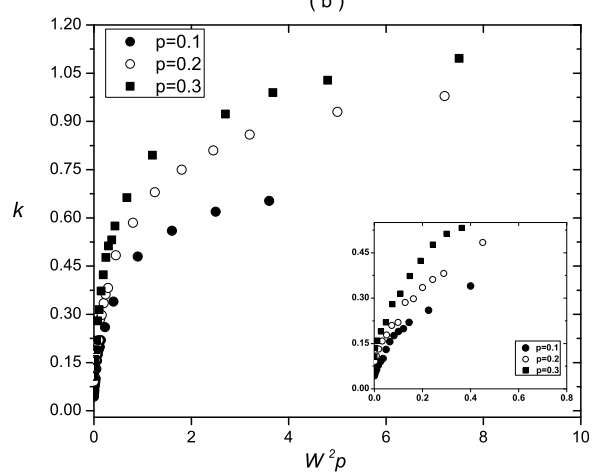

(c)

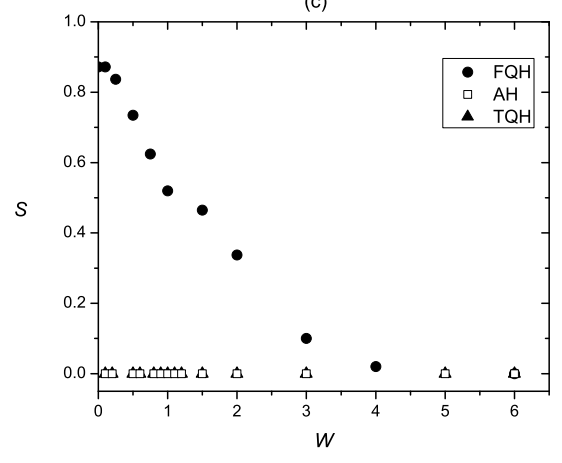

FIG. 10. Dependence of the size $k=\xi^{-1}$ of Larkin-Imry-Ma domains, obtained by fitting simulation $G(r)$ to the exponentially decaying form given by eq.(14). In both plots, $T=0.5, L=70$.

(a) $\xi(W)$ alone, for different values of $p$; (b) (TQH only); $\xi\left(W^{2} p\right)$ (see discussion in text), (c) $S(W)$, where $S=\sqrt{b_{0}}$ (see Eq.(14)).

dependent properties. The RAN [49] and SSS [61] RF Lebwohl-Lasher lattice models introduced to study liquid crystals in porous media have exhibited many interesting properties. Detailed studies of history dependence, however, which explicitly pertain to glass properties, have not so far been carried out. Accordingly, therefore, this study has focused on the influence of system history on long-time steady state properties.

The steady states which the Brownian dynamics provides for our RF systems sometimes correspond to thermal equilibrium, and sometimes to metastable states in which the nematic spins are trapped even after relatively long simulation runs. In our simulations, we have distinguished temperature quenched history (TQH), field quenched history (FQH), and annealed history $(\mathrm{AH})$. TQH and FQH represent two limiting extremes encountered in typical experimental studies.

In TQH we quench a randomly orientationally distributed nematic spin configurations (corresponding to $T \rightarrow \infty)$ to a low temperature $T \ll T^{*}$, and then allow the system to equilibrate, insofar as it is able. In FQH, by contrast, we allow a set of aligned nematic spins to relax. The field (of FQH) here is implicitly assumed sufficiently large for the nematic spins to be initially completely aligned. In AH the temperature was reduced slowly. The procedure involved reducing temperature in a step-wise fashion from a high temperature $T \gg T^{*}$ and allowing dynamical relaxation. The initial configuration at a given temperature is a configuration obtained from a previous (slightly higher) temperature. The majority of our simulations involved TQH and FQH, which folklore suggests are less likely to give rise to true thermal equilibria, and are correspondingly more likely to involve restricted dynamical manifolds. However, some AH simulations were also carried out. We note that this procedure is thought to broaden the manifold of dynamically accessible states [77], or in informal language, to favor thermal equilibria.

From the calculated steady-state configuration manifolds, we have extracted two-point correlation functions $G(r)$, and local and global orientational order parameter. Finite-size scaling methods [73, 78] have then been used to distinguish phases with LRO, QLRO and SRO. In principle, discrimination between the different types of correlation functions could also be inferred by fitting $G(r)$ to the model forms given in eqs.(14),(15) corresponding to each of these regimes. In practice, however, we have found in our simulations that finite-size scaling analyses yield more robust results (see also [49]).

We have simulated system at relatively low temperatures (at $T \sim T_{N I} / 2$ ) where local degree of order is substantial over a range of impurity-nematic interaction parameters $W \in] 0,4]$, and concentrations $p \in] 0, p_{\max }=$ 0.3]. The maximum concentration $p_{\max }<p_{c} \sim 0.31$, where $p_{c}$ is the percolation threshold.

The main finding of our simulations are as follows. For TQH LRO, QLRO or SRO seem all to occur. We note that in random field systems such as those studied in this paper, LRO is forbidden as an equilibrium phase by the Larkin-Imry-Ma argument. However, a quench in a random system restricts the manifold of accessible states, and thus the theorem does not apply. It is possible, however, that in dilute impurity systems such as ours, finite size effects mimic LRO. Resolution of this point awaits further study.

However, we can say that for FQH, the SRO regime is absent. However, the definition of the phases depends both on an analysis of the correlation function, and on a finite-size scaling analysis of order parameters in different simulations. As a consequence, the crossover between regimes is difficult to define precisely. We have there- 
fore only been able to estimate crossover values of $W$ separating LRO-QLRO $\left(W=W_{Q L R O}^{(\text {history })}\right)$ and QLRO$\operatorname{SRO}\left(W=W_{S R O}^{(T Q H)}\right)$ regimes. For $p=0.1$ we obtain $W_{Q L R O}^{(T Q H)} \sim W_{Q L R O}^{(F Q H)} \sim 0.1 \pm 0.05$ and $W_{S R O}^{(T Q H)} \sim 3 \pm 0.2$.

Within structures exhibiting prevailing QLRO character a domain-type structural pattern is imprinted, as first suggested by Giamarchi and Doussal [76]. In the case of QLRO the structural crossover between neighboring domains is on average smoother in comparison to the SRO case. For both histories estimated characteristic domain sizes $\xi$ are comparable. In the weak coupling regime Larkin-Imry-Ma scaling $\xi \sim D^{-2 /(4-d)}$ is roughly obeyed. Our simulations roughly support our mean-field estimate suggesting $D(d=3) \sim \sqrt{p} W$. In the temperature-annealed history simulations that we have carried out, we obtain structures the characteristics of which are closer to TQH case.

In summary, our study reveals that in systems of our interest initial conditions could significantly influence even macroscopic properties of structures. The LIM domain-type pattern is imprinted in weak enough coupling regime both for QLRO and SRO structures.

The systems of our study bear at least some connections with several experimental systems. These are either LCs confined to various porous matrices, or binary mixtures of LCs with nano- or colloidal particles. In the former case RF-type disorder is produced by essentially random spatial variations of LC-pore interfaces. In the case of binary mixtures RF-like disorder could be established even in case of spherical particles of radius $R$ which by themselves do not enforce any preferred direction in space. However, for strong enough anchoring $\left(R / d_{e}>1\right.$ (where $d_{e}$ stands for the surface extrapolation length), they could locally enforce a preferred direction in a low-temperature phase. For example, for strong enough homeotropic anchoring they might locally enforce dipolar, quadrupolar or even more complex symmetry [79].

In future work we intend to establish closer analogies with magnetic systems. In magnetic systems, the termi- nology describing different glass-like structures is rather well-established. By analogy with the term 'speromagnetic', one commonly refers to a phase with nematic lowtemperature SRO domain patterns as a 'speronematic' phase [80]. However, the related speromagnetic phase [81] refers to cases of relatively strong coupling with RF in which the local magnetic moments point essentially along a local RF easy axis. A closer magnetic analog is correlated spin glass, where short range correlations extend over a coherence length that is much larger than a typical intermolecular distance. QLRO nematic structures are more reminiscent of ferromagnets with wandering axis [81]. In particular, we intend to study in detail external field loop hysteresis behavior in nonergodic regimes. We note that recent studies reveal that pinning of topological line defects in RF-type systems can play a significant role [63]. However, nematic and magnetic systems differ significantly with respect to line defect topology.

\section{ACKNOWLEDGMENTS}

TJS acknowledges a generous visiting Government of Slovenia, Ministry of Education professorship at the Faculty of Physics, University of Maribor over the period October-December 2011, when this work was started. He further acknowledges the hospitality of SK and of Professor N. Vaupotič (Dean of the Faculty) over this period. SK and TJS acknowledge the hospitality of the Isaac Newton Insitute for Mathematical Sciences, Cambridge, UK, where both were programme visitors in the 2013 "Mathematics of Liquid Crystals" programme, and where this work was completed. TJS thanks R. Pelcovits (Brown), C. Zannoni (Bologna) and particularly P. Shukla (NEHU, Shillong, India), for helpful discussions. SK acknowledges the support of the European Office of Aerospace Research and Development Grant FA8655-121-2068. A.R. acknowledges the support of a Slovenian Research Agency (ARRS) young researcher grant.
[1] J. S. Rowlinson and B. Widom, Molecular theory of capillarity (Clarendon Press, 1982).

[2] T. F. Meister and D. M. Kroll, Phys. Rev. A 31, 4055 (1985).

[3] M. E. Fisher and M. N. Barber, Phys. Rev. Lett. 28, 1516 (1972).

[4] P. Sheng, Phys. Rev. Lett. 37, 1059 (1976).

[5] P. Sheng, Phys. Rev. A 26, 1610 (1982).

[6] A. Poniewierski and T. J. Sluckin, Mol. Cryst. Liq. Cryst. 111, 373 (1984).

[7] M. M. T. da Gama, Molec. Phys. 53, 611 (1984).

[8] A. Poniewierski and T. J. Sluckin, Mol. Cryst. Liq. Cryst. 126, 143 (1985).

[9] T. J. Sluckin, Mol. Cryst. Liq. Cryst. 179, 349 (1990).
[10] M. D. Dadmun and M. Muthukumar, J. Chem. Phys. 98, doi:10.3762/bjoc.6.2 (1993).

[11] R. J. Ondris-Crawford, G. P. Crawford, S. Žumer, and J. W. Doane, Phys. Rev. Lett. 70, 194 (1993).

[12] G. P. Crawford and S. Zumer, eds., Liquid crystals in complex geometries formed by polymer and porous networks (Taylor and Francis, 1996).

[13] C. Cramaer, T. Cramer, F. kremer, and R. Stannarius, J. Chem. Phys. 106, 3730 (1996).

[14] C. Fehr, C. Goze-Bac, E. Anglaret, C. Benoit, and A. Hasmy, EPL 73, 553 (2006).

[15] T. Jin and D. Finotello, Phys. Rev. E 69, 041704 (2004).

[16] S. Tripathi, C. Rosenblatt, and F. M. Aliev, Phys. Rev. Lett. 72, 2725 (1994). 
[17] M. Buscaglia, T. Bellini, V. Degiorgio, F. Mantegazza, and F. Simoni, EPL 48, 634 (1999).

[18] X.-. Wu, W. I. Goldburg, M. X. Liu, and J. Z. Xue, Phys. Rev. Lett. 69, 470 (1992).

[19] T. Bellini, N. A. Clark, L. Wu, C. W. Garland, D. Schaefer, and B. Olivier, Phys. Rev. Lett. 69, 788 (1992).

[20] G. S. Iannacchione, G. P. Crawford, S. Zumer, J. W. Doane, and D. Finotello, Phys. Rev. Lett. 71, 245506 (1993).

[21] T. Bellini, N. A. Clark, and D. W. Schaefer, Phys. Rev. Lett. 74, 2740 (1995)

[22] M. Buscaglia, T. Bellini, C. Chiccoli, F. Mantegazza, P. Pasini, M. Rotunno, and C. Zannoni, Phys. Rev. E 74, 011706 (2006).

[23] G. S. Iannacchione and D. Finotello, Phys. Rev. Lett. 69, 2595 (1992).

[24] G. S. Iannacchione and D. Finotello, Liq. Cryst. 14, 1135 (1993).

[25] M. Caggioni, A. Roshi, S. Barjami, F. Mantegazza, G. S. Iannacchione, and T. Bellini, Phys. Rev. Lett. 93, 127801 (2004)

[26] M. Marinelli, F. Mercuri, S. Paoloni, and U. Zammit, Phys. Rev. Lett. 95, 237801 (2005).

[27] Z. Kutnjak, S. Kralj, G. Lahajnar, and S. Žumer, Phys. Rev. E 68, 021705 (2003).

[28] Z. Kutnjak, S. Kralj, G. Lahajnar, and S. Žumer, Phys. Rev. E 70, 51703 (2004).

[29] Z. Kutnjak, S. Kralj, and S. Žumer, Phys. Rev. E 66, 041702 (2002)

[30] G. Cordoyiannis, G. Nounesis, V. Bobnar, S. Kralj, and Z. Kutnjak, Phys. Rev. Lett. 94, 027801 (2005).

[31] G. Cordoyiannis, S. Kralj, G. Nounesis, S. Žumer, and Z. Kutnjak, Phys. Rev. E 73, 031707 (2006).

[32] G. Cordoyiannis, S. Kralj, G. Nounesis, Z. Kutnjak, and S. Žumer, Phys. Rev. E 75, 021702 (2007).

[33] A. Shahin, Y. M. Joshi, and S. A. Ramakrishna, Langmuir 27, 1404514052 (2011).

[34] R. Harris, M. J. Plischke, and M. J. Zuckermann, Phys. Rev. Lett. 31, 160 (1973).

[35] M. Mézard, G. Parisi, and M. A. Virasoro, Spin glass theory and beyond (World Scientific, 1987).

[36] A. I. Larkin, Soviet Phys, JETP 31, 784 (1970).

[37] Y. Imry and S. K. Ma, Phys. Rev. Lett. 35, 1399 (1975).

[38] E. M. Chudnovsky, J. Appl. Phys. 64, 5770 (1988).

[39] R. A. Pelcovits, E. Pytte, and J. Rudnick, Phys. Rev. Lett. 40, 476 (1978).

[40] A. Aharony and E. Pytte, Phys. Rev. Lett. 45, 1583 (1980).

[41] E. Callen, Y. J. Liu, and J. R. Cullen, Phys. Rev. B. 81, 263 (1977).

[42] D. R. Denholm and T. J. Sluckin, Phys. Rev. B 48, 901 (1993).

[43] P. A. Lebwohl and G. Lasher, Phys. Rev. A 6, 426 (1972).

[44] U. Fabbri and C. Zannoni, Molec. Phys. 58, 763 (1986).

[45] W. Maier and A. Saupe, Z. Naturforsch. 13a, 564 (1958).

[46] H. Zink and W. H. de Jeu, Mol. Cryst. Liq. Cryst. 124, 287 (1985).

[47] S. V. Fridrikh and E. M. Terentjev, Phys. Rev. Lett. 79, 4661 (1997)

[48] S. V. Fridrikh and E. M. Terentjev, Phys. Rev. E 60, 1847 (1999).

[49] D. J. Cleaver, S. Kralj, T. J. Sluckin, and M. P. Allen, "The random anisotropy nematic spin model," in Liquid crystals in complex geometries formed by polymer and porous networks, edited by G. P. Crawford and S. Zumer (Taylor and Francis, 1996) Chap. 21.

[50] V. Popa-Nita, Eur. J. Phys. B 12, 83 (1999).

[51] S. Kralj and V. Popa-Nita, Eur. J. Phys. E 14, 115 (2004).

[52] S. Kralj, V. Popa-Nita, and M. Svetec, "Random anisotropy liquid crystal model," in Phase transitions. applications to liquid crystals, organic electronic and optoelectronic fields, edited by V. Popa-Nita (Research Signpost, 2006) Chap. 4.

[53] D. E. Feldman, Phys. Rev. Lett. 84, 4886 (2000).

[54] D. E. Feldman, Int. J. Mod. Phys. B 15, 2945 (2001).

[55] D. E. Feldman and R. A. Pelcovits, Phys. Rev. E 70, 040702(R) (2004).

[56] B. M. Khasanov, Magnetic Resonance in solids 6, 95 (2004).

[57] Z. Zhang and A. Chakrabarti, EPL 33, 23 (1996).

[58] J. Chakrabarti, Phys. Rev. Lett. 81, 1847 (1998).

[59] V. Popa-Nita and S. Kralj, Phys. Rev. E. 73, 041705 (2006).

[60] A. Ranjkesh, M. Ambrožič, G. Cordoyiannis, Z. Kutnjak, and S. Kralj, Advances in Condensed Matter Physics 2013, 505219 (2013).

[61] T. Bellini, M. Buscaglia, C. Chiccoli, F. Mantegazza, P. Pasini, and C. Zannoni, Phys. Rev. Lett. 85, 1008 (2000).

[62] T. Bellini, M. Buscaglia, C. Chiccoli, F. Mantegazza, P. Pasini, and C. Zannoni, Phys. Rev. Lett. 88, 245506 (2002).

[63] M. Rotunno, M. Buscaglia, C. Chiccoli, F. Mantegazza, P. Pasini, T. Bellini, and C. Zannoni, Phys. Rev. Lett. 94, 097802 (2005).

[64] J. M. Fish and R. L. C. Vink, Phys. Rev. Lett. 105, 147801 (2010).

[65] D. R. Denholm, Simulation studies of random-anisotropy magnets, Ph.D. thesis, University of Southampton (1995).

[66] R. Fisch, Phys. Rev. B 58, 5684 (1998).

[67] D. P. Landau, "Monte Carlo studies of finite size effects at first and second order phase transitions," in Finite size scaling and numerical simulation of statistical systems, edited by V. Privman (World Scientific, 1990) Chap. 5.

[68] Z. Bradač, S. Kralj, and S.Žumer, Phys. Rev. E 58, 7447 (1998).

[69] D. L. Ermak and J. A. McCammon, J. Chem. Phys. 69, 1352 (1978).

[70] E. Dickinson, S. A. Allison, and J. A. McCammon, J. Chem. Soc. Fraday Trans. 2 81, 591 (1985).

[71] R. Ying and M. H. Peters, J. Chem. Phys. 95, 1234 (1991).

[72] M. H. Peters and R. Ying, J. Chem. Phys. 98, 6492 (1993).

[73] R. Eppenga and D. Frenkel, Mol. Phys. 52, 1303 (1984).

[74] Z. Bradač, S. Kralj, and S.Žumer, Phys. Rev. E 34, 17 (2011).

[75] E. F. Gramsbergen, L. Longa, and W. H. de Jeu, Phys. Rep. 135, 195 (1986).

[76] T. Giamarchi and P. L. Doussal, Phys. Rev. B 52, 1242 (1995).

[77] S. Kirkpatrick, C. D. Gelatt, and M. P. Vecchi, Science 220, 671 (1983).

[78] K. Binder and D. W. Heermann, Monte Carlo Simulation in Statistical Physics; An Introduction. (Springer, 1988) Chap. 2.3. 
[79] P. Poulin, V. Cabuil, and D. A. Weitz, Phys. Rev. Lett. 862 (1997).

[80] V. Popa-Nita and S. Romano, Chem. Phys. 264, 91 (2001), and references therein.
[81] E. Chudnovsky, W. Saslow, and R. Serota, Phys. Rev. B 33, 251 (1986). 